\title{
Digital License Plate Detection and Recognition with Improved Vertical Edge Detection and Template Matching
}

\author{
A F M Shahab Uddin ${ }^{1}$, Feroza Naznin ${ }^{2}$, Md. Alamgir Hossain ${ }^{3}$, Md. Zahidul Islam ${ }^{4}$ \\ Research Asst, Dept of Information and Communication Engineering, Islamic University, Kushtia, Bangladesh 1,2 \\ Assistant Professor, Dept of Information and Communication Engineering, Islamic University, Kushtia, Bangladesh ${ }^{3}$ \\ Professor, Dept of Information and Communication Engineering, Islamic University, Kushtia, Bangladesh ${ }^{4}$
}

\begin{abstract}
Digital License Plate Detection and Recognition is one of the most common and important technology for the intelligent world, where our trend is to automate everything. Now a days, Digital License Plate Detection and Recognition is widely used in traffic control, toll-pay, real-time monitoring and parking systems in many developed countries. Different standards and styles for license plate exist all over the world. So there is a clear absence of standardization of number plate. Therefore it is hard to detect and recognize all of them by a single algorithm. The techniques used in this project are Vertical Edge Detection with Ratio Verifying method for number plate detection and Template Matching for license number recognition. Vertical Edge Detection is performed for detecting the possible plate regions and Ratio of each region is verified for extracting the actual number plate. After the detection process, the detected plate is further processed for recognizing the license number. Recognition is carried out by segmentation and Template Matching algorithm. The new system is examined for several car images and is capable of recognizing various license numbers at a satisfactory level.
\end{abstract}

Keywords: License plate recognition, improved template matching, character recognition, Pattern Recognition, computer vision.

\section{INTRODUCTION}

Number of vehicles is increasing rapidly to satisfy the needs of large number of population over the world. This large number of vehicles increasing the difficulties of enforcing the law and traffic rules manually. Some places in the road have some speed limits and the speed of vehicles passing through the road must be kept below the limits. Traffic signals are placed in several places over the city and every vehicle must follow these signals. Private car parking lot and toll-collecting booth are installed, where the cars have to pay the toll and parking fees. So, each of these systems has one thing in common, that is the vehicles.

The primary and most important task to automate these processes is to identify these vehicles. And it is obvious that, recognizing the license plate of these vehicles is the only way to identify them. Every vehicle must have a unique license number, which is written on the license number plate attached in front and rear of the vehicle. This license number gives the identity to the vehicle. The automated systems have the task of capturing the vehicles and detecting its number plate and extracting its license number from the number plate.

Once the license number is recognized, information about the owner of that vehicle can easily be retrieved from the database.Before recognizing the characters, the number plate location must be identified from the captured image and the region needs to be segmented. This is the most important part of Digital License Plate Detection and Recognition, because the accuracy of the result is mostly depended on it.

Since there is a clear absence of standardization of license number plate and other disturbances like poor image quality, different plate background, different plate and character size make the detection process very difficult.

\section{REVIEW OF OTHER TECHNIQUES}

In recent years several methods have been proposed in order to improve the process of automatic detection and recognition of license plates with their own advantages and disadvantages.

Shan $\mathrm{Du}[1]$ proposed a system whichuses some features, such as the boundary, the color, or the existence of the characters for plate detection and neural networks and fuzzy classifiers for license number recognition.J. Arrospide [2] designed a descriptor based on log-Gabor functions for vehicle verification instead of state-of-the-art descriptors based on Gabor functions.A. M. Al-Ghaili [3]proposed a fast vertical edge detection algorithm based on the contrast between the grayscale values. Hao Chen [4] used auto-correlation curve, projection properties and 
Vol. 6, Issue 1, January 2017

character position feature on a binary image. These Plate Detection includes Vertical Edge Detection and methods are applied in combination to detect the actual Ratio Verifying Technique based on the license plate license plate. Gisu Hero [5] developed license plate model. In addition, Morphological and Flood-fill operation detection technique using group of lines forming rectangle are performed to increase the efficiency.

at the plate boundary. SerkanOzbay and Ergun Ercelebi[6] developed smearing algorithm to locate the license plate. The License number Recognition includes Connected Mei Yu and Yong Deak Kim [7] proposed vertical edge Component Analysis to segment the characters and detection followed by size, shape filter for edge area and Template Matching algorithm for recognizing these edge matchingtechnique based on plate model. characters.

FarhadFaradji, Amir HosseinRezaie and MajidZiaratban [8] used Sobel edge detection, vertical projection analysis to locate plate area. Compact factor is used to remove false plate area. License number in the detected license plates is segmented based on projection analysis, Hough transform and region growing. Xiangjian $\mathrm{He}$, WenjingJia, BijanSamali, LihongZheng, Qiang $\mathrm{Wu}$ and MarimuthuPalaniswami [9] proposed a method which uses horizontal and vertical projection analysis for character segmentation. Yuangang Zhang and Changshui Zhang [10] developed character segmentation using Hough Transform. Horizontal edge detection using Hough Transform is applied to segment the characters. Feng Yang, Zheng Ma and Mei Xie [11] proposed region growing algorithm to segment the characters.

\section{PROPOSED TECHNIQUE}

The proposed method is categorized into two sections; the first is "Plate Detection" and the second is "License number Recognition".

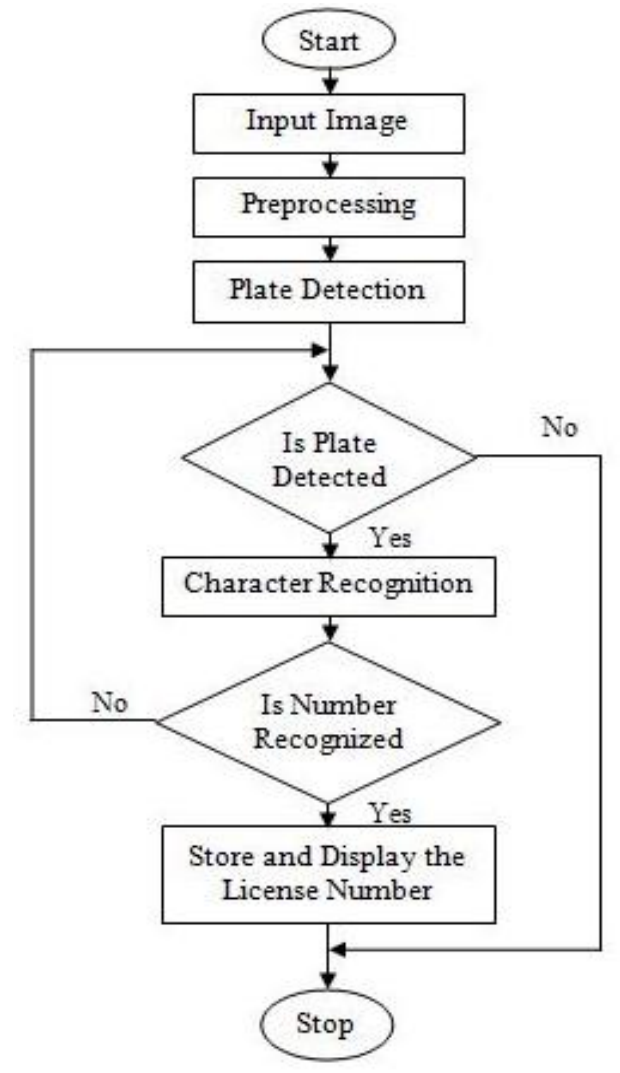

Fig.1. Block diagram of the proposed system

\section{NUMBER PLATE DETECTION}

A License Plate must have a high number of vertical edges. We have used this feature to locate the plate. In order to proceed with this consideration we have converted the image into greyscale image because colour image is not suitable for this task. At the same time noise has been removed by using a Gaussian blur of 5 x 5 .

\section{TABLE I ALGORITHM FOR PLATE DETECTION TECHNIQUE}

[1] Load car image

[2] Pre-processing

a. Convert to Greyscale image

b. Noise reduction by Gaussian Blur operation

[3] Vertical Edge detection using Sobel filter

[4] Morphological Close operation

[5] Find all possible regions using Connected Component analysis.

[6] Verify the ratio of each regions and eliminate unwanted regions

[7] Perform Flood-fill operation

[8] Remove unwanted angles

[9] Extract possible Number Plate

[10] Stop

We have used Sobel filter to detect vertical edges. After that, Threshold filter is applied to obtain a binary image with a threshold value resulted through Otsu's method. By applying a close morphological operation, blank spaces between each vertical edge line are removed and connected all regions that have a high number of edges.

After that we have the possible regions that can contain plates. However, most of the regions will not contain license plates. Contours of these regions are created and for each contour, the Area and Aspect-ratio is analysed. The region whose aspect ratio is close to $450 / 100=4.5$ (plate width divided by plate height) with an error margin of $40 \%$ and which is based on a minimum of 15 pixels and maximum of 125 pixels for the height, must be the plate.All of these plates may not have the same size and lighting condition. So we need to resize these plates to a standard format andapply Histogram Equalization operation to take care of the lighting condition. The resulted plate image is sent to the Recognition Part for further processing. 
Vol. 6, Issue 1, January 2017

\section{CHARACTER RECOGNITION}

\section{A. Character Segmentation}

Prior to license number recognition, all characters must be segmented first. Character segmentation has the task of selecting each character as individual image. We can segment each character using connected component analysis. For each region, we can make a size verification and remove all regions where the size is smaller or the aspect is not correct. We will assume an area as a region of black pixels if it is higher than $80 \%$ and can't be a character.If a segmented region passes the verification process, we have to set the same size and position for all characters.

\section{B. Template Matching}

The objective of this section is to recognize each segmented characters. Recognition includes scanning and analysingevery characters and converting them into digital format which can be used by the computer.

\section{TABLE II ALGORITHM FOR TEMPLATE MATCHING TECHNIQUE}

For $\mathrm{i}=0: \mathrm{n}$, where $\mathrm{n}$ is the number of segmented characters

[1] Load seg_char[i]

[2] for $\mathrm{j}=0: \mathrm{m}$, where $\mathrm{m}$ is the number of template characters

[3] Load temp_char[j]

[4] Perform seg_char[i] $\bigoplus$ temp_char[j]

[5] Count nonzero elements

[6] Store the value into Distance vector D

[7] Increase j

[8] Find the position of the minimum value of $\mathrm{D}$

[9] Using the position, retrieve the recognized character

[10] Add the character to a string name License Number

[11] Increase i

[12] Show the License Number

[13] Stop

To recognize the characters, template matching technique is used in this project. Template for each alphanumeric character is created previously. Then for each segmented character, the Exclusive-OR operation is performed with every template characters we have. This gives the pixel difference between the two characters. The minimum difference is identified. And for which template character, the minimum difference is identified, is the recognized character. We can represent the template matching process as by the following mathematical expression:

$$
\text { Distance Vector } \mathrm{D}_{\mathrm{k}}=\sum \sum\left(\mathrm{X}_{\mathrm{ij}} \oplus \mathrm{Y}_{\mathrm{ij}}\right)
$$

Where $\mathrm{D}$ is a distance vector of $1 \times 36$ (Because we have 36 template character) dimension, whose elements represent the distance from the template character to the detected character.
Now we can find the minimum distance among all of the elements and trace its position within the distance vector D.

$$
\text { Detected Character Position } \mathrm{P}=\min (\mathrm{D})
$$

Where $\mathrm{P}$ is the position of the detected character. Since we have the position of the detected character, we can easily recognize that character.

$$
\text { Recognized Character } \mathrm{C}=\text { Character }[\mathrm{P}]
$$

Where Character is a vector of same dimension as D and has all the alpha-numeric characters. This process is performed for each of the segmented character and the recognized characters are stored as string.

\section{RESULT}

The proposed method for License Plate Detection and Recognition has been tested on several car images. The system is simulated in CodeBlock 10.05 on Core i5 1.70 $\mathrm{GHz}$ PC. The images were taken from:

- Different locations, where the background is nonuniform.

- Different lighting conditions.

- Different angles.

- Different size of the images

TABLE III PERFORMANCE MEASUREMENT OF THE PROPOSED TECHNIQUE

\begin{tabular}{|l|c|c|c|c|}
\hline Unit & $\begin{array}{l}\text { No. of } \\
\text { Car }\end{array}$ & $\begin{array}{l}\text { Succ } \\
\text { ess }\end{array}$ & $\begin{array}{l}\text { Accura } \\
\text { cy }\end{array}$ & $\begin{array}{l}\text { Executi } \\
\text { on Time }\end{array}$ \\
\hline Detection & 105 & 96 & $91.43 \%$ & $140 \mathrm{~ms}$ \\
\hline Recognition & 96 & 91 & $94.79 \%$ & $141 \mathrm{~ms}$ \\
\hline Total & 105 & 91 & $86.67 \%$ & $281 \mathrm{~ms}$ \\
\hline
\end{tabular}

Total 105 images were taken from different location as well as different conditions. The result of the proposed License Plate Detection and Recognition system is shown in Table II. The proposed method successfully detected 96 license plates among 105 car images and was unable to detect license plate from 9 images. So the accuracy for license plate detection is $91.43 \%$.

Then the detected 96 plates were sent to the recognition system, where template matching algorithm is used. Among these96 plates, 91 license numbers were successfully recognized and the system failed to recognize 5 plate images. So the accuracy for license number recognition is $94.79 \%$.

Finally, the system was able to successfully recognizing 91 license numbers among 105 car images. So the overall accuracy of the system is $86.67 \%$.

The execution time taken for number plate detection is 140 millisecond and for license number recognition is 141 millisecond. The total execution time taken by the proposed system is 281 millisecond.Some of the results are shown in Fig 2. 
Vol. 6, Issue 1, January 2017

\section{CONCLUSION AND FUTURE WORK}

In this project our proposed a method for Digital License Plate Detection and Recognition is divided into two main functional module. The first is License Plate Detection using Vertical edge detection and Ratio analysis. And the second is the License Number Recognition which is further subdivided into two section including Character Segmentation using Connected Component Analysis and Character Recognition using Template Matching Algorithm.
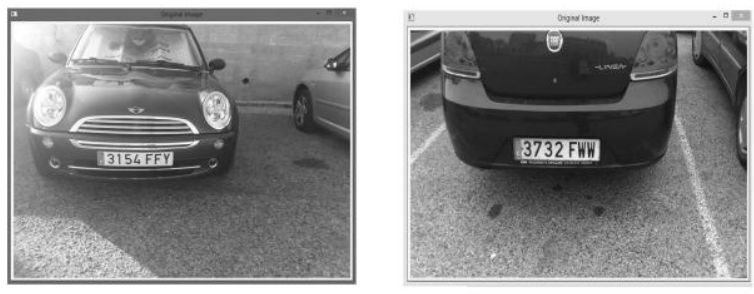

(a)
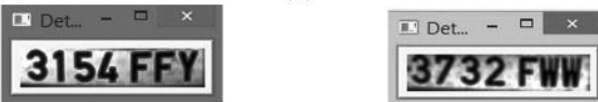

3154FFY

3732FWW (c)

Fig.2.(a) Captured Image (b) Detected Plate and (c) Recognized License Number

Although the proposed technique is developed for specific format number plate, I believe that this technique can be implemented worldwide. Mostly the detection of the number plate can be implemented easily. Also the same recognition of license number can be applicable anywhere by only changing the templates stored in the database.

The future challenge of this research is to make the system fast enough to work in real-time.

\section{ACKNOWLEDGMENT}

The authors gratefully acknowledge the assistance of Dr. Md. Zahidul Islam, Director of Computer Vision and Intelligent Interfacing lab (CVIIL), Dept. of Information and Communication Engineering, Islamic University, Kushtia, Bangladesh, for his helpful guideline.

\section{REFERENCES}

[1] M. Ibrahim and M. Shehata, "Automatic license plate recognition (ALPR): A state-of-art review," IEEE Transaction on Circuits and Systems for Video Technology, Vol. 23, NO. 2, February 2013.

[2] J. Arrospide and L. Salgado, "Log-gabor filters for image-based vehicle verification," IEEE Transaction on Image Processing, Vol. 22, No. 6, June 2013.

[3] A. M. Al-Ghaili, S. Mashohor, A. R. Ramli and A. Ismail, "Vertical edge-based car license plate detection method," IEEE, Manuscript Received January 25,2012.

[4] Hao Chen, JishengRen, Huachun Tan, Jianqun Wang, " A novel method for license plate localization”, 4th Proc. of ICIG 2007, pp. 604-609.

[5] GisuHeo, Minwoo Kim, Insook Jung, DukRyongLee,IlSeok Oh, "Extraction of car license plate regions using line grouping and edge density methods", International Symposium on Information Technology Convergence, 2007, pp. 37-42.

[6] SerkanOzbay, Ergun Ercelebi, "Automatic vehicle identification by plate recognition”, Proc. of PWASET, vol. 9, no. 4, 2005, pp. 222225.

17] Mei Yu and Yong Deak Kim, "An approach to Korean license plate recognition based on vertical edge matching", IEEE International Conference on System, Man and Cybernetics, 2000, vol.4, pp. 2975-2980.

[8] Farhad Faradji, Amir Hossein Rezaie, Majid Ziaratban, "A Morphological based License Plate Location", ICIP, 2007, pp. I 57I 60.

[9] Xiangjian He et al, "Segmentation of characters on car license plates", 10th Workshop on Multimedia Signal Processing, Oct. 2008, pp. 399-402.

[10] Yungang Zhang, Changshui Zhang, "A New algorithm for character segmentation of license plate", Proc. Of IEEE Intelligent Vehicles Symposium, 2003, pp. 106-109.

[11] Feng Yang, Zheng Ma, Mei Xie, "A Novel approach for license plate character segmentation", ICIEA, 2006, pp.1-6.

\section{BIOGRAPHIES}

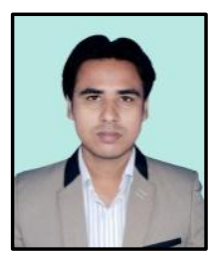

AFM Shahab Uddin was born in Chuadanga, Bangladesh in 1989.He received the B.Sc. degree in Information and Communication Engineering (ICE) from Islamic University, Kushtia, Bangladesh in 2013. He is currently studying toward the M.Sc. degree at the same department.As present he is a research assistant in Computer Vision \& Intelligent Interfacing Lab (CVIIL) at the department of ICE, Islamic University, Kushtia, Bangladesh. His research interests include computer vision, human-robot interaction and artificial neural networks.

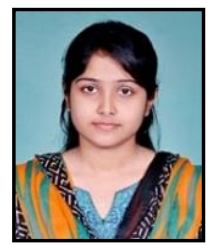

Feroza Naznin has completed her B.Sc. and M.Sc. from the Dept. of Information and Communication Engineering, Islamic University, Kushtia, Bangladesh in 2014 and 2015 respectively. Currently she is a research assistant in Computer Vision \& Intelligent Interfacing Lab (CVIIL) of Department of Information \& Communication Engineering, Islamic University, Kushtia, Bangladesh. Her research interests are artificial neural network, Computer Vision, Image processing etc.

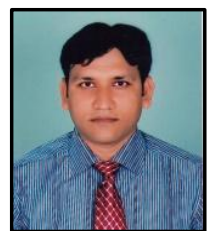

Md. Alamgir Hossain received the Bachelor's and Master's Degree in the Dept. of Information and Communication Engineering (ICE) from Islamic University, Kushtia, in 2003 and 2004, respectively. He is currently an assistant Professor in the department of ICE, Islamic University, Kushtia-7003, and Bangladesh. He was a lecturer in the Dept. of Computer Science \& Engineering from Institute of Science Trade \&Technology (Under National University), Dhaka, Bangladesh, from 23th October,2007 to 18th April 2010. Since 2012, he has been working as a Researcher at the Computer Vision \& Intelligent Interfacing Lab (CVIIL), Department of ICE, Islamic 
University, Kushtia. Hiscurrent research interests include Computer Vision, Machine Learning and IoT.

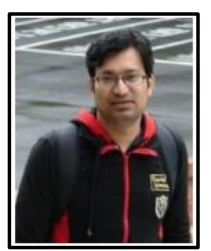

Md. Zahidul Islam has received his B.Sc. and M.Sc. degree from the Department of Applied Physics and Electronic Engineering, University of Rajshahi (RU), Bangladesh in 2000 and 2002 respectively. In 2003, he has joined as a Lecturer in the Department of Information \& Communication Engineering, Islamic University (IU), Kushtia, Bangladesh. He has done his $\mathrm{Ph} . \mathrm{D}$ research on Visual Object Tracking System from the Department of Computer Engineering at Intelligent Image Media and Interface Lab, Chonnam National University (CNU), South Korea. In August 2011, Dr. Islam has been successfully awarded his $\mathrm{PhD}$ from the same department. Besides, he has done his research internship in 3D Vision Lab in Samsung Advanced Institute of Technology (SAIT), Suwon, South Korea. Dr. Islam has also other research interests like computer vision, 3D object, human and motion tracking and tracking articulated body and genetic algorithm etc. Currently he is a Professor in the Department of Information \& Communication Engineering, Islamic University (IU), Kushtia, Bangladesh. Also he is the director of Computer Vision \& Intelligent Interfacing Lab (CVIIL) and Coordinator of ICE Innovation Lab at the same department. 